\title{
The Aging Heart: Mitophagy at the Center of Rejuvenation
}

\author{
Wenjing J. Liang and Åsa B. Gustafsson* \\ Department of Pharmacology, Department of Medicine, Skaggs School of Pharmacy and Pharmaceutical Sciences, \\ University of California, San Diego, CA, United States
}

\section{OPEN ACCESS}

Edited by:

Junichi Sadoshima,

University of Medicine and Dentistry of New Jersey, United States

Reviewed by:

Satoaki Matoba

Kyoto Prefectural University of

Medicine, Japan

Qiangrong Liang,

New York Institute of Technology,

United States

Satoru Kobayashi,

New York Institute of Technology,

United States

*Correspondence:

Åsa B. Gustafsson

abgustafsson@ucsd.edu

Specialty section:

This article was submitted to

Cardiovascular Metabolism,

a section of the journal

Frontiers in Cardiovascular Medicine

Received: 07 November 2019

Accepted: 03 February 2020

Published: 19 February 2020

Citation:

Liang WJ and Gustafsson $\AA B$ (2020)

The Aging Heart: Mitophagy at the

Center of Rejuvenation.

Front. Cardiovasc. Med. 7:18.

doi: 10.3389/fcvm.2020.00018
Aging is associated with structural and functional changes in the heart and is a major risk factor in developing cardiovascular disease. Many recent studies have focused on increasing our understanding of the basis of aging at the cellular and molecular levels in various tissues, including the heart. It is known that there is an age-related decline in cellular quality control pathways such as autophagy and mitophagy, which leads to accumulation of potentially harmful cellular components in cardiac myocytes. There is evidence that diminished autophagy and mitophagy accelerate the aging process, while enhancement preserves cardiac homeostasis and extends life span. Here, we review the current knowledge of autophagy and mitophagy in aging and discuss how age-associated alterations in these processes contribute to cardiac aging and age-related cardiovascular diseases.

Keywords: aging, autophagy, mitophagy, mitochondria, heart, PINK1, Parkin, mitophagy receptors

\section{INTRODUCTION}

Aging is a major risk factor in developing cardiovascular disease and increases exponentially with age. Cardiac aging is characterized by the presence of hypertrophy, fibrosis, accumulation of misfolded proteins, and dysfunctional mitochondria. Current efforts are dedicated to understanding the biological process of aging and to identify pathways that can be targeted to extend health and life spans. Interestingly, it has been demonstrated that many of the pathways that improve health and extend longevity in various organisms all converge on autophagy (1-8). Autophagy is a catabolic pathway that is responsible for recycling cellular proteins and organelles to maintain energy homeostasis. It participates in the elimination of pathogens and prevents activation of inflammation. It is also a key pathway in cellular quality control by eliminating dysfunctional or unwanted organelles and protein aggregates. However, there is strong evidence that autophagy is decreased with age in tissues, including the heart $(5,9-15)$.

The heart requires a lot of energy which is mainly generated by mitochondria via oxidative phosphorylation. However, aging is associated with altered cardiac mitochondrial metabolism and mitochondrial respiratory defects (16). The impaired fatty acid and glucose metabolism, combined with reduced mitochondrial respiration are also believed to underlie the increased susceptibility to cardiac injury in the elderly population (16). Normally, these dysfunctional mitochondria are eliminated by autophagosomes in a selective process termed mitophagy. Predictably, reduced autophagy in aging contributes to accumulation of dysfunctional mitochondria and decreased ability to adapt to stress.

Altered autophagy and mitophagy overtime are likely central contributors in the aging process. Here, we review the current knowledge of autophagy and mitophagy in aging and discuss how age-associated alterations in these processes contribute to cardiac aging and age-related cardiovascular diseases. 


\section{AUTOPHAGY}

Autophagy involves the sequestration of ubiquitinated cargo into vesicles called autophagosomes and delivery of the content to lysosomes via fusion. The cargo is degraded inside lysosomes and the components are recycled to the cytoplasm. Autophagy is a highly regulated process and consists of several distinct steps; initiation, nucleation and formation of phagophore, sequestration of cargo, and fusion of autophagosome with a lysosome (Figure 1A). The different steps in the process are regulated by different autophagy-related proteins (Atg) (17). The mechanistic target of rapamycin (mTOR) functions as a gate keeper and prevents activation of autophagy. When mTOR is inhibited, it leads to activation of the unc-51 like autophagy activating kinase 1 (Ulk1/Atg1) which initiates the nucleation of the autophagosome via Beclin1 (18). At baseline, Beclin1 is sequestered by $\mathrm{Bcl}-2$ and Rubicon to suppress autophagy but its release allows it to initiate autophagosome formation (19-21). The elongation and maturation of the growing autophagosome membrane requires two conjugation pathways. The E1-like and E2-like enzymes Atg7 and Atg10 conjugate Atg5 to Atg12. The Atg5-Atg12 complex then interacts with Atg16. Atg16 is required for the proper localization of the complex to the pre-autophagosomal membrane (22). The
Atg5-12-16 complex then functions as an E3-like enzyme in the second conjugation pathway, where LC3 is covalently linked to phosphatidylethanolamine (PE). The conjugation of LC3 to PE to form LC3II is mediated by Atg7 (E1-like) and Atg3 (E2-like), respectively (17). LC3II is also involved in cargo recognition where it binds to adaptor proteins such as p62 (23). Several proteins in this pathway are altered with age which ultimately leads to diminished autophagy.

\section{MITOPHAGY}

\section{PINK1/Parkin-Mediated Mitophagy}

The PINK1/Parkin pathway contains three key elements: a mitochondrial membrane depolarization sensor (PINK1), a signal amplifier (Parkin) and a downstream signal effector (ubiquitin chains) (Figure 1B) (24). Under normal cellular conditions, PINK1 is partly imported into the inter mitochondrial membrane space where it is cleaved by resident proteases such as the presenilin-associated rhomboid-like protease (PARL) $(25,26)$. However, this process is disrupted upon loss of mitochondrial membrane potential, leading to accumulation of PINK1 on the outer mitochondrial membrane (OMM), where PINK1 in turn recruits the E3 ubiquitin ligase Parkin $(25,27,28)$. PINK1 phosphorylates both ubiquitin and

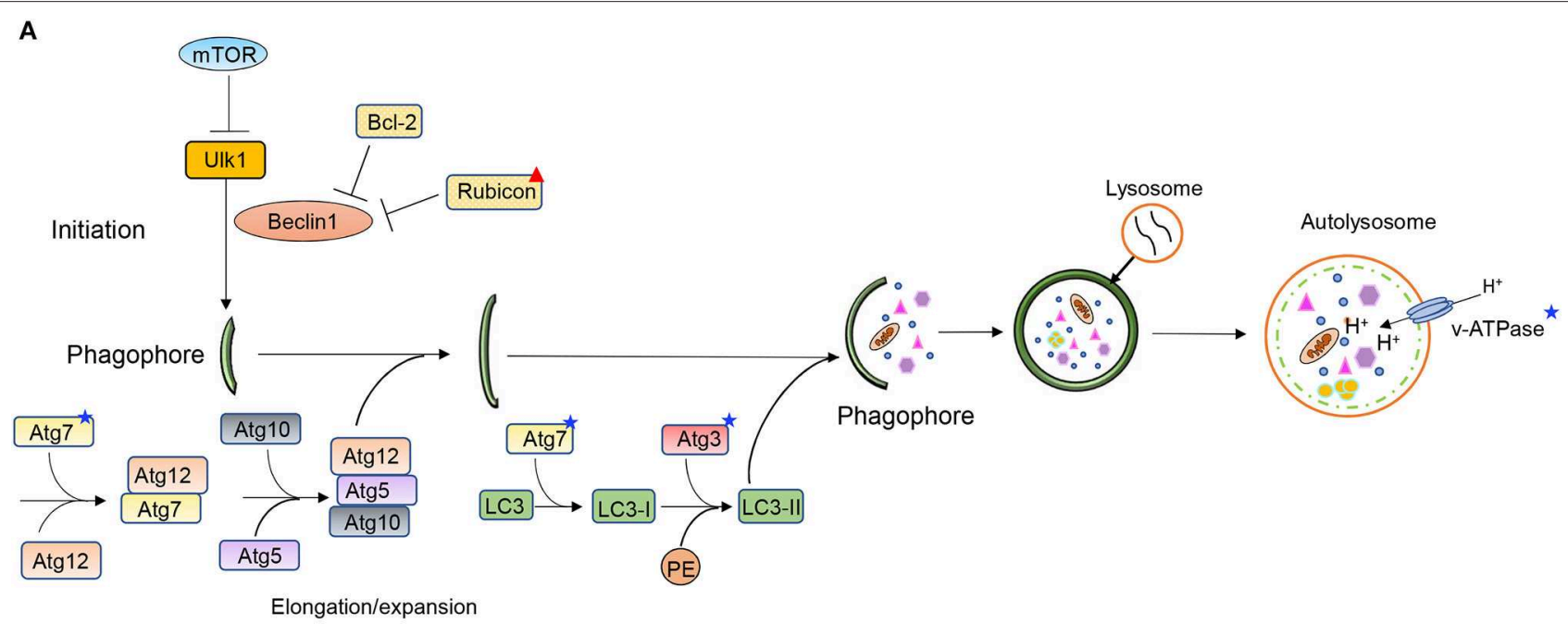

B

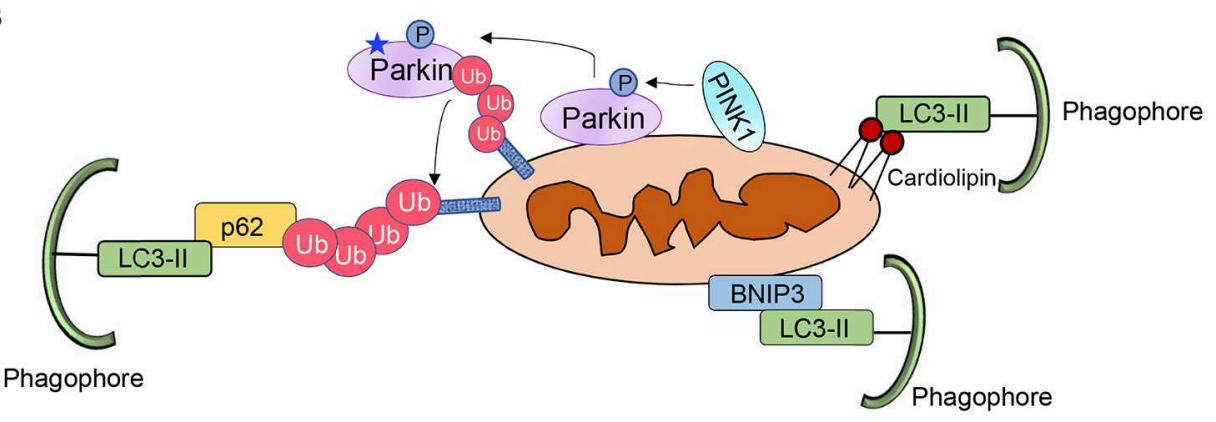

FIGURE 1 | Overview of (A) autophagy and (B) mitophagy pathways. Stars and triangle mark proteins that have been reported to be altered with age. 
Parkin which contribute to both its activation and anchoring at the mitochondria (29). PINK1 has also been reported to phosphorylate MFN2 which then functions as a docking site for Parkin at mitochondria (30). This allows activated Parkin to ubiquitinate various outer mitochondrial membrane proteins (31). However, a recent study reported an alternative function for MFN2 during mitophagy where MFN2 must be degraded for mitophagy to proceed (32). MFN2 is known to tether mitochondria to ER at specific contact sites. McLelland et al. found that Parkin-mediated ubiquitination and degradation of MFN2 disrupts the contact sites and releases mitochondria from the ER. The release provides Parkin full access to its other substrates and allows for mitophagy to proceed (32). The mitochondrial proteins ubiquitinated by Parkin are recognized by various adaptor proteins, such as p62/SQSTM1 and Optineurin $(33,34)$. These adaptors bind to the ubiquitinchains on proteins in the OMM via their ubiquitin-associated (UBA) domain and simultaneously directly interact with LC3 on the autophagosome via their LC3 Interacting Region (LIR) motifs $(23,33,35)$.

\section{Mitophagy Receptors}

Mitochondrial proteins in the OMM can also target mitochondria to autophagosomes (Figure 1B). BNIP3, NIX/BNIP3L, FUNDC1, Bcl2L13, FKBP8, and Prohibitin-2 (PHB2) are some of the mitophagy receptors that have been identified to date (36-41). These proteins are integrated mitochondrial membrane proteins that are facing the cytosol. The exception is PHB2, which is localized in the inner mitochondrial membrane. PHB2 promotes removal of remaining mitochondrion after outer membrane rupture (36). The mitophagy receptors contain LIRs and can therefore bind directly to LC3 on the autophagosome membrane bypassing the need for ubiquitin and adaptor proteins. The phospholipid cardiolipin can also function as a mitophagy receptor (Figure 1B). Cardiolipin is localized on the inner mitochondrial membrane but is externalized on dysfunctional mitochondria where it facilitates mitophagy by interacting with LC3 (42). However, it is possible that, similar to PHB2, cardiolipin can ensure mitophagy of the inner mitochondrial compartment after outer mitochondrial membrane rupture. Although they have all been established as mitophagy receptors, it is unclear how most of them are activated to induce mitophagy of mitochondria. These proteins are also known to have alternative functions and how they switch between the two functions is not completely clear.

The physiological conditions dictating activation of the two distinct mitophagy pathways are still unclear and under intense investigation. Recently, it has been proposed that PINK1/Parkinmediated mitophagy plays a minimal role in basal mitophagy $(43,44)$ and that this pathway plays a more important role in stress adaptation and repair $(45,46)$. Other studies have reported that mitophagy receptors are key regulators of programmed mitophagy during development or differentiation (47-49). Thus, the two different mitophagy pathways appear to have distinct functions in the cell but additional studies are clearly needed. Moreover, cross talk clearly exists between the two mitophagy pathways $(50,51)$. For instance, the protein phosphatase PGAM5 dephosphorylates FUNDC1 which enhances the interaction between FUNDC1 and LC3 (52). PGAM5 also coordinates with PHB2 to promote PINK1/Parkin-mediated mitophagy where PHB2 decreases PINK1 processing by inhibiting PARL while PGAM5 stabilizes PINK1 on the OMM (53). Taken together, there is clearly coordination between these two pathways, and they can compensate for each other to some extent.

\section{AUTOPHAGY AND AGING}

A growing body of data support the anti-aging effects of enhanced autophagy. Many studies have demonstrated that enhancing autophagy by limiting caloric intake, genetic manipulation or pharmacological treatments increases lifespan in various organisms (1-6). For instance, transgenic mice with systemic overexpression of Atg5 have enhanced autophagic activity in tissues which leads to health benefits such as reduced weight gain with age and extended life spans compared to wild type mice (2). Although this study did not specifically focus on the myocardium, the authors reported increased autophagic activity as well as reduced fibrosis with age in hearts of the transgenic mice. The cardioprotective effects of enhanced autophagy during the aging process were recently confirmed by the Levine group, who developed a Becn1 ${ }^{F 121 A / F 121 A}$ knock-in mouse model with constitutively increased basal autophagy due to a disruption in the Bcl-2 binding to Beclin1. They found that health and life spans are significantly increased in the knockin mice. Moreover, aged Becn1 $1^{F 121 A / F 121 A}$ knock-in mice have reduced cardiac hypertrophy and interstitial fibrosis compared to aged-matched wild type mice (20), confirming that preserving autophagy in the heart delays or even prevents cardiac aging. In contrast, selective disruption of autophagy in the heart leads to accelerated cardiac aging with accumulation of ubiquitinated proteins and dysfunctional mitochondria and development of cardiac hypertrophy (54). Preserving autophagy is clearly critical in the heart to prevent biological aging.

\section{MITOPHAGY AND AGING}

Reduced mitophagy also recapitulates the age-related accumulation of dysfunctional mitochondria in tissues. Thus, the forced increase in autophagy in the above studies can also be linked to enhanced mitophagy as it would enhance elimination of dysfunctional mitochondria. Several studies have confirmed that genetic and pharmacological interventions promoting enhanced mitophagy also lead to extended life span $(55,56)$, while disrupting mitophagy leads to accelerated aging phenotypes $(57,58)$. For instance, Urolithin A is a natural compound that induces mitophagy and extends life span in C.elegans (56). Both systemic and neuron-specific overexpression of Parkin in flies slows aging and extends lifespan, although lifespan extension is greater with ubiquitous Parkin overexpression (59). A link also exists between Parkin-mediated mitophagy and NLRP3 inflammasome activation. The NLRP3 inflammasome is activated by the presence of mitochondrial DNA in the 
cytosol that have been released from damaged mitochondria. Thus, Parkin-mediated mitophagy of damaged mitochondria functions to prevent activation of the inflammasome (60). The PINK1/Parkin pathway also diminishes STING-induced inflammation by a similar mechanism (61).

Several early studies reported that PINK1 or Parkin deficiency in Drosophila causes accumulation of dysfunctional mitochondria, flight muscle degeneration and reduced lifespan (62-64). Also, Cornelissen et al. found that mitophagic activity in flight muscle increased with aging in flies and that the age-dependent rise is abrogated by either PINK1 or Parkin deficiency (57). Parkin-deficient mice have an accelerated aging phenotype and accumulate aberrant mitochondria in aging heart $(58,65)$ while cardiac specific overexpression of Parkin can delay cardiac aging by enhancing mitochondrial turnover (65). These studies present evidence that enhancing mitophagy by targeting the Parkin pathway is beneficial. However, the antiaging effect of Parkin is likely dose-dependent as aged transgenic mice with higher levels of Parkin overexpression develop cardiac fibrosis likely due to an imbalance between ubiquitination and autophagic degradation (66).

Much less is known about what happens to mitophagy receptors during aging. It was recently reported that mice deficient in both Akt2 and AMPK are predisposed to cardiac aging possible due to compromised mitophagy. These hearts have reduced levels of several mitophagy proteins including BNIP3 and FUNDC1 (15). A mouse model carrying a proofreadingdefective mtDNA polymerase $\gamma$ (POLG) accumulate mtDNA mutations which leads to accelerated aging (67). Unexpectedly, Parkin plays a minimal role in clearing cardiac mitochondria in POLG mice as cardiac aging is unaffected by cardiacspecific overexpression or global deletion of Parkin (66). Instead, hearts in aged POLG mice have elevated levels of the mitophagy receptor BNIP3 coupled with enhanced mitochondrial biogenesis, indicating enhanced baseline mitochondrial turnover (66). The fact that NIX/BNIP3 double knockout mice accumulate dysfunctional mitochondria in the heart at an accelerated rate with age compared to wild type mice confirms that these mitophagy receptors play a key role in baseline mitochondrial maintenance (68). Furthermore, Rana et al. recently demonstrated that promoting Drp1-mediated mitochondrial fission in midlife leads to increased mitophagy and rejuvenated mitochondria in flies. This leads to improved health span and delays the onset of pathology linked to aging (69). Together, these findings support the notion that reduced mitophagy might be a significant underlying factor in the accumulation of dysfunctional mitochondria in aged organisms contributing to their health decline and mortality. Also, the mitophagy pathway may represent a therapeutic target to counteract aging.

\section{AGE-RELATED REDUCTION IN AUTOPHAGY AND MITOPHAGY}

Although autophagy is clearly diminished with age in tissues, including the heart $(5,9-12)$, exactly why cardiac autophagy is reduced during aging is still unclear. Most of our current knowledge comes from studies in cell lines or other tissues. Oxidative stress can inhibit autophagy by promoting oxidation of the autophagy enzymes involved in autophagy (70). Under baseline conditions when autophagy is not activated, LC3 is covalently bound to inactive Atg3 and Atg7, which protects cysteine residues in their catalytic sites from oxidation. However, the release of LC3 upon activation of autophagy leads to exposure of the cysteines, making them available to direct oxidation during high levels of oxidative stress (70). Moreover, Parkin is also prone to oxidation of its cysteine residues which affects its E3 ubiquitin ligase activity and promotes its misfolding and aggregation $(71,72)$. Also, both PINK1 and Parkin can be S-nitrosylated which leads to attenuated mitophagy (73, 74). As cardiac aging is characterized by increased oxidative stress $(75,76)$, it is possible that this directly contributes to reduced autophagosome formation and impaired Parkinmediated mitophagy in aged myocytes.

Low levels of chronic inflammation has also been linked to age-related diseases (77). The NLRP3 inflammasome is a cytosolic protein complex that initiates activation of inflammatory responses by inducing cell death and triggering the release of proinflammatory cytokines (77). Deregulation of the NLRP3 inflammasome has been linked to inhibition of autophagy and aging. NLRP3-deficient mice have improved health span and attenuated age-related functional decline, including reduced bone loss, improved memory and cognitive performance, and motor performance (78). Recently, it was reported that aged NLRP3-deficient mice have reduced cardiac hypertrophy and fibrosis and increased life spans compared to wild type mice (14). This study linked the NLRP3-deficiency in aged mice to reduced mTOR suppression resulting in increased autophagic activity (14).

Moreover, it is also likely that proteins involved in regulating autophagy are altered with age. For instance, Rubicon is a negative regulator of Beclin1 and it was recently reported that Rubicon expression increases in worm, fly and mouse tissues with age (5). Rubicon knockdown ameliorates age-dependent phenotypes and extends life span in both worms and flies, while Rubicon systemic-knockout mice have reduced age-associated phenotypes such as decreased kidney fibrosis (5). This suggests that Rubicon could be one of the factors contributing to the decline in autophagy during aging. However, other regulators might also be altered with age in tissues.

Finally, lysosomes function in the terminal step of autophagy (Figure 1) and lysosomal function is compromised with age (79). For instance, the activity of lysosomal hydrolases responsible for degrading cargo is dependent on the acidic milieu of the lysosome. After fusion with an autophagosome, the lysosome must undergo reacidification to restore the acidic $\mathrm{pH}$ and activate the hydrolases. The v-type ATPase is responsible for maintaining the acidic milieu by pumping proton into the lysosomal lumen and studies indicate that the v-ATPase activity and acidification are reduced with age (80). Lysosomal dysfunction has been identified in age-related neurological pathologies, such as Parkinson's and Alzheimer's disease (80). Lysosomal impairment has also been associated with decreased lifespan, 
while enhancing lysosomal functional capacity can promote longevity $(81,82)$. In addition, the adult brain contains a pool of neural stem cells (NSCs) that can generate new neurons but the function of NSCs declines with age. Interestingly, there is an age-dependent decrease in lysosome levels in NSCs which results in fewer lysosomes available to fuse with autophagosomes (83). It is currently unclear if lysosomal function is altered in the aged heart.

\section{CONCLUSION}

In summary, declines in autophagy and mitophagy in tissues clearly play a role in the aging process and contribute to development of age-related diseases. The main questions that remain unanswered include: why are autophagy and mitophagy suppressed with age and can these pathways be restored in the aged heart? Relatively little is still known about the molecular mechanism underlying the decrease in autophagy and mitophagy and whether there are tissue specific differences.

\section{REFERENCES}

1. Eisenberg T, Abdellatif M, Schroeder S, Primessnig U, Stekovic S, Pendl $\mathrm{T}$, et al. Cardioprotection and lifespan extension by the natural polyamine spermidine. Nat Med. (2016) 22:1428-38. doi: 10.1038/nm.4222

2. Pyo JO, Yoo SM, Ahn HH, Nah J, Hong SH, Kam TI, et al. Overexpression of Atg5 in mice activates autophagy and extends lifespan. Nat Commun. (2013) 4:2300. doi: $10.1038 /$ ncomms3300

3. Fernandez AF, Sebti S, Wei Y, Zou Z, Shi M, McMillan KL, et al. Disruption of the beclin 1-BCL2 autophagy regulatory complex promotes longevity in mice. Nature. (2018) 558:136-40. doi: 10.1038/s41586-018-0162-7

4. Toth ML, Sigmond T, Borsos E, Barna J, Erdelyi P, Takacs-Vellai K, et al. Longevity pathways converge on autophagy genes to regulate life span in Caenorhabditis elegans. Autophagy. (2008) 4:330-8. doi: 10.4161/auto.5618

5. Nakamura S, Oba M, Suzuki M, Takahashi A, Yamamuro T, Fujiwara M, et al. Suppression of autophagic activity by Rubicon is a signature of aging. Nat Commun. (2019) 10:847. doi: 10.1038/s41467-019-08729-6

6. Simonsen, Cumming RC, Brech A, Isakson P, Schubert DR, Finley KD. Promoting basal levels of autophagy in the nervous system enhances longevity and oxidant resistance in adult Drosophila. Autophagy. (2008) 4:176-84. doi: 10.4161/auto.5269

7. Harrison DE, Strong R, Sharp ZD, Nelson JF, Astle CM, Flurkey K, et al. Rapamycin fed late in life extends lifespan in genetically heterogeneous mice. Nature. (2009) 460:392-5. doi: 10.1038/nature08221

8. Wilkinson JE, Burmeister L, Brooks SV, Chan CC, Friedline S, Harrison DE, et al. Rapamycin slows aging in mice. Aging Cell. (2012) 11:675-82. doi: 10.1111/j.1474-9726.2012.00832.x

9. Inuzuka Y, Okuda J, Kawashima T, Kato T, Niizuma S, Tamaki $\mathrm{Y}$, et al. Suppression of phosphoinositide 3-kinase prevents cardiac aging in mice. Circulation. (2009) 120:1695-703. doi: 10.1161/CIRCULATIONAHA.109.871137

10. Taneike M, Yamaguchi O, Nakai A, Hikoso S, Takeda T, Mizote I, et al. Inhibition of autophagy in the heart induces age-related cardiomyopathy. Autophagy. (2010) 6:600-6. doi: 10.4161/auto.6.5.11947

11. Ren X, Chen L, Xie J, Zhang Z, Dong G, Liang J, et al. Resveratrol ameliorates mitochondrial elongation via Drp1/Parkin/PINK1 signaling in senescent-like cardiomyocytes. Oxid Med Cell Longev. (2017) 2017:4175353. doi: 10.1155/2017/4175353

12. Zhou J, Chong SY, Lim A, Singh BK, Sinha RA, Salmon AB, et al. Changes in macroautophagy, chaperone-mediated autophagy, and mitochondrial metabolism in murine skeletal and cardiac muscle during aging. Aging. (2017) 9:583-99. doi: 10.18632/aging.101181
Although manipulation of autophagy and mitophagy pathways are protective in pre-clinical models, the level of activity must be carefully monitored as excessive autophagy can lead to excessive degradation of key cellular components. Increased knowledge into how these pathways are regulated as well as altered with age will allow for more specific manipulation. Further understanding will also provide important insights into how future therapies can protect the heart against age-specific functional decline.

\section{AUTHOR CONTRIBUTIONS}

Both authors contributed to the content of this article and have approved of its submission.

\section{FUNDING}

$\AA ̊$ G is supported by NIH R01HL138560 and R01HL132300, and TRDRP 27IR-0013 and 28IP- 0025. WL is supported by TRDRP T30FT0846.
13. Ren J, Yang L, Zhu L, Xu X, Ceylan AF, Guo W, et al. Akt2 ablation prolongs life span and improves myocardial contractile function with adaptive cardiac remodeling: role of Sirt1-mediated autophagy regulation. Aging Cell. (2017) 16:976-87. doi: 10.1111/acel.12616

14. Marin-Aguilar F, Lechuga-Vieco AV, Alcocer-Gomez E, Castejon-Vega B, Lucas J, Garrido C, et al. NLRP3 inflammasome suppression improves longevity and prevents cardiac aging in male mice. Aging Cell. (2019) 19:e13050. doi: 10.1111/acel.13050

15. Wang S, Kandadi MR, Ren J. Double knockout of Akt2 and AMPK predisposes cardiac aging without affecting lifespan: role of autophagy and mitophagy. Biochim Biophys Acta Mol Basis Dis. (2019) 1865:1865-75. doi: 10.1016/j.bbadis.2018.08.011

16. Lesnefsky EJ, Chen Q, Hoppel CL. Mitochondrial metabolism in aging heart. Circ Res. (2016) 118:1593-611. doi: 10.1161/CIRCRESAHA.116.3 07505

17. Levine B, Kroemer G. Biological functions of autophagy genes: a disease perspective. Cell. (2019) 176:11-42. doi: 10.1016/j.cell.2018.09.048

18. Kim J, Kundu M, Viollet B, Guan KL. AMPK and mTOR regulate autophagy through direct phosphorylation of Ulk1. Nat Cell Biol. (2011) 13:132-41. doi: $10.1038 / \mathrm{ncb} 2152$

19. Matsunaga K, Saitoh T, Tabata K, Omori H, Satoh T, Kurotori N, et al. Two Beclin 1-binding proteins, Atg14L and Rubicon, reciprocally regulate autophagy at different stages. Nat Cell Biol. (2009) 11:385-96. doi: $10.1038 /$ ncb1846

20. Zhong Y, Wang QJ, Li X, Yan Y, Backer JM, Chait BT, et al. Distinct regulation of autophagic activity by Atg14L and Rubicon associated with Beclin 1-phosphatidylinositol-3-kinase complex. Nat Cell Biol. (2009) 11:46876. doi: $10.1038 /$ ncb1854

21. Pattingre S, Tassa A, Qu X, Garuti R, Liang XH, Mizushima N, et al. Bcl2 antiapoptotic proteins inhibit Beclin 1-dependent autophagy. Cell. (2005) 122:927-39. doi: 10.1016/j.cell.2005.07.002

22. Fujita N, Itoh T, Omori H, Fukuda M, Noda T, Yoshimori T. The Atg16L complex specifies the site of LC3 lipidation for membrane biogenesis in autophagy. Mol Biol Cell. (2008) 19:2092-100. doi: 10.1091/mbc.e07-1 2-1257

23. Pankiv S, Clausen TH, Lamark T, Brech A, Bruun JA, Outzen H, et al. p62/SQSTM1 binds directly to Atg8/LC3 to facilitate degradation of ubiquitinated protein aggregates by autophagy. J Biol Chem. (2007) 282:24131-45. doi: 10.1074/jbc.M702824200

24. Harper JW, Ordureau A, Heo JM. Building and decoding ubiquitin chains for mitophagy. Nat Rev Mol Cell Biol. (2018) 19:93-108. doi: $10.1038 / \mathrm{nrm} .2017 .129$ 
25. Jin SM, Lazarou M, Wang C, Kane LA, Narendra DP, Youle RJ. Mitochondrial membrane potential regulates PINK1 import and proteolytic destabilization by PARL. J Cell Biol. (2010) 191:933-42. doi: 10.1083/jcb.201008084

26. Greene AW, Grenier K, Aguileta MA, Muise S, Farazifard R, Haque $\mathrm{ME}$, et al. Mitochondrial processing peptidase regulates PINK1 processing, import and Parkin recruitment. EMBO Rep. (2012) 13:378-85. doi: 10.1038/embor.2012.14

27. Matsuda N, Sato S, Shiba K, Okatsu K, Saisho K, Gautier CA, et al. PINK1 stabilized by mitochondrial depolarization recruits Parkin to damaged mitochondria and activates latent Parkin for mitophagy. J Cell Biol. (2010) 189:211-21. doi: 10.1083/jcb.200910140

28. Narendra D, Tanaka A, Suen DF, Youle RJ. Parkin is recruited selectively to impaired mitochondria and promotes their autophagy. J Cell Biol. (2008) 183:795-803. doi: 10.1083/jcb.200809125

29. Kane LA, Lazarou M, Fogel AI, Li Y, Yamano K, Sarraf SA, et al. PINK1 phosphorylates ubiquitin to activate Parkin E3 ubiquitin ligase activity. J Cell Biol. (2014) 205:143-53. doi: 10.1083/jcb.201402104

30. Chen Y, Dorn GW 2nd. PINK1-phosphorylated mitofusin 2 is a Parkin receptor for culling damaged mitochondria. Science. (2013) 340:471-5. doi: 10.1126/science. 1231031

31. Sarraf SA, Raman M, Guarani-Pereira V, Sowa ME, Huttlin EL, Gygi SP, et al. Landscape of the PARKIN-dependent ubiquitylome in response to mitochondrial depolarization. Nature. (2013) 496:372-6. doi: 10.1038/nature12043

32. McLelland GL, Goiran T, Yi W, Dorval G, Chen CX, Lauinger ND, et al. Mfn2 ubiquitination by PINK1/parkin gates the p97-dependent release of ER from mitochondria to drive mitophagy. Elife. (2018) 7:e32866. doi: 10.7554/eLife.32866

33. Lazarou M, Sliter DA, Kane LA, Sarraf SA, Wang C, Burman JL, et al. The ubiquitin kinase PINK1 recruits autophagy receptors to induce mitophagy. Nature. (2015) 524:309-14. doi: 10.1038/nature14893

34. Narendra D, Kane LA, Hauser DN, Fearnley IM, Youle RJ. p62/SQSTM1 is required for Parkin-induced mitochondrial clustering but not mitophagy; VDAC1 is dispensable for both. Autophagy. (2010) 6:1090-106. doi: 10.4161/auto.6.8.13426

35. Geisler S, Holmstrom KM, Skujat D, Fiesel FC, Rothfuss OC, Kahle PJ, et al. PINK1/Parkin-mediated mitophagy is dependent on VDAC1 and p62/SQSTM1. Nat Cell Biol. (2010) 12:119-31. doi: 10.1038/ncb2012

36. Wei Y, Chiang WC, Sumpter R Jr, Mishra P, Levine B. Prohibitin 2 is an inner mitochondrial membrane mitophagy receptor. Cell. (2017) 168:224-38 e10. doi: 10.1016/j.cell.2016.11.042

37. Bhujabal Z, Birgisdottir AB, Sjottem E, Brenne HB, Overvatn A, Habisov S, et al. FKBP8 recruits LC3A to mediate Parkin-independent mitophagy. EMBO Rep. (2017) 18:947-961. doi: 10.15252/embr.201643147

38. Murakawa T, Yamaguchi O, Hashimoto A, Hikoso S, Takeda T, Oka T, et al. Bcl-2-like protein 13 is a mammalian Atg32 homologue that mediates mitophagy and mitochondrial fragmentation. Nat Commun. (2015) 6:7527. doi: $10.1038 /$ ncomms 8527

39. Liu L, Feng D, Chen G, Chen M, Zheng Q, Song P, et al. Mitochondrial outer-membrane protein FUNDC1 mediates hypoxia-induced mitophagy in mammalian cells. Nat Cell Biol. (2012) 14:177-85. doi: 10.1038/ncb2422

40. Novak I, Kirkin V, McEwan DG, Zhang J, Wild P, Rozenknop A, et al. Nix is a selective autophagy receptor for mitochondrial clearance. EMBO Rep. (2010) 11:45-51. doi: 10.1038/embor.2009.256

41. Hanna RA, Quinsay MN, Orogo AM, Giang K, Rikka S, Gustafsson AB. Microtubule-associated protein 1 light chain 3 (LC3) interacts with Bnip3 protein to selectively remove endoplasmic reticulum and mitochondria via autophagy. J Biol Chem. (2012) 287:19094-104. doi: 10.1074/jbc.M111.3 22933

42. Chu CT, Ji J, Dagda RK, Jiang JF, Tyurina YY, Kapralov AA, et al. Cardiolipin externalization to the outer mitochondrial membrane acts as an elimination signal for mitophagy in neuronal cells. Nat Cell Biol. (2013) 15:1197-205. doi: $10.1038 /$ ncb2837

43. McWilliams TG, Prescott AR, Montava-Garriga L, Ball G, Singh F, Barini E, et al. Basal mitophagy occurs independently of PINK1 in mouse tissues of high metabolic demand. Cell Metab. (2018) 27:439-49 e5. doi: 10.1016/j.cmet.2017.12.008
44. Lee JJ, Sanchez-Martinez A, Zarate AM, Beninca C, Mayor U, Clague MJ, et al. Basal mitophagy is widespread in Drosophila but minimally affected by loss of Pink1 or parkin. J Cell Biol. (2018) 217:1613-22. doi: 10.1083/jcb.201801044

45. Kubli DA, Zhang X, Lee Y, Hanna RA, Quinsay MN, Nguyen CK, et al. Parkin protein deficiency exacerbates cardiac injury and reduces survival following myocardial infarction. J Biol Chem. (2013) 288:915-26. doi: 10.1074/jbc.M112.411363

46. Song M, Gong G, Burelle Y, Gustafsson AB, Kitsis RN, Matkovich $\mathrm{SJ}$, et al. Interdependence of parkin-mediated mitophagy and mitochondrial fission in adult mouse hearts. Circ Res. (2015) 117:346-51. doi: 10.1161/CIRCRESAHA.117.306859

47. Lampert MA, Orogo AM, Najor RH, Hammerling BC, Leon LJ, Wang BJ, et al. BNIP3L/NIX and FUNDC1-mediated mitophagy is required for mitochondrial network remodeling during cardiac progenitor cell differentiation. Autophagy. (2019) 15:1182-98. doi: 10.1080/15548627.2019.1580095

48. Esteban-Martinez L, Sierra-Filardi E, McGreal RS, Salazar-Roa M, Marino G, Seco E, et al. Programmed mitophagy is essential for the glycolytic switch during cell differentiation. EMBO J. (2017) 36:1688-706. doi: 10.15252/embj.201695916

49. Sandoval H, Thiagarajan P, Dasgupta SK, Schumacher A, Prchal JT, Chen M, et al. Essential role for Nix in autophagic maturation of erythroid cells. Nature. (2008) 454:232-5. doi: 10.1038/nature07006

50. Zhang T, Xue L, Li L, Tang C, Wan Z, Wang R, et al. BNIP3 protein suppresses PINK1 kinase proteolytic cleavage to promote mitophagy. J Biol Chem. (2016) 291:21616-29. doi: 10.1074/jbc.M116.733410

51. Gao F, Chen D, Si J, Hu Q, Qin Z, Fang M, et al. The mitochondrial protein BNIP3L is the substrate of PARK2 and mediates mitophagy in PINK1/PARK2 pathway. Hum Mol Genet. (2015) 24:2528-38. doi: 10.1093/hmg/ddv017

52. Chen G, Han Z, Feng D, Chen Y, Chen L, Wu H, et al. A regulatory signaling loop comprising the PGAM5 phosphatase and CK2 controls receptor-mediated mitophagy. Mol Cell. (2014) 54:362-77. doi: 10.1016/j.molcel.2014.02.034

53. Yan C, Gong L, Chen L, Xu M, Abou-Hamdan H, Tang M, et al. PHB2 (prohibitin 2) promotes PINK1-PRKN/Parkin-dependent mitophagy by the PARL-PGAM5-PINK1 axis. Autophagy. (2019) 16:419-34. doi: 10.1080/15548627.2019.1628520

54. Nakai A, Yamaguchi O, Takeda T, Higuchi Y, Hikoso S, Taniike M, et al. The role of autophagy in cardiomyocytes in the basal state and in response to hemodynamic stress. Nat Med. (2007) 13:619-24. doi: 10.1038/nm1574

55. Schiavi, Maglioni S, Palikaras K, Shaik A, Strappazzon F, Brinkmann V, Torgovnick A, et al. Iron-starvation-induced mitophagy mediates lifespan extension upon mitochondrial stress in C. elegans. Curr Biol. (2015) 25:181022. doi: $10.1016 /$ j.cub.2015.05.059

56. Ryu D, Mouchiroud L, Andreux PA, Katsyuba E, Moullan N, Nicolet-DitFelix AA, et al. Urolithin A induces mitophagy and prolongs lifespan in $C$. elegans and increases muscle function in rodents. Nat Med. (2016) 22:879-88. doi: $10.1038 / \mathrm{nm} .4132$

57. Cornelissen T, Vilain S, Vints K, Gounko N, Verstreken P, Vandenberghe W. Deficiency of parkin and PINK1 impairs age-dependent mitophagy in Drosophila. Elife. (2018) 7:e35878. doi: 10.7554/eLife.35878

58. Kubli DA, Quinsay MN, Gustafsson AB. Parkin deficiency results in accumulation of abnormal mitochondria in aging myocytes. Commun Integr Biol. (2013) 6:e24511. doi: 10.4161/cib.24511

59. Rana A, Rera M, Walker DW. Parkin overexpression during aging reduces proteotoxicity, alters mitochondrial dynamics, and extends lifespan. Proc Natl Acad Sci USA. (2013) 110:8638-43. doi: 10.1073/pnas.1216197110

60. Zhong Z, Umemura A, Sanchez-Lopez E, Liang S, Shalapour S, Wong J, et al. NF- $\kappa B$ restricts inflammasome activation via elimination of damaged mitochondria. Cell. (2016) 164:896-910. doi: 10.1016/j.cell.2015.12.057

61. Sliter DA, Martinez J, Hao L, Chen X, Sun N, Fischer TD, et al. Parkin and PINK1 mitigate STING-induced inflammation. Nature. (2018) 561:258-62. doi: 10.1038/s41586-018-0448-9

62. Greene JC, Whitworth AJ, Kuo I, Andrews LA, Feany MB, Pallanck LJ. Mitochondrial pathology and apoptotic muscle degeneration in Drosophila parkin mutants. Proc Natl Acad Sci USA. (2003) 100:4078-83. doi: $10.1073 /$ pnas. 0737556100 
63. Clark IE, Dodson MW, Jiang C, Cao JH, Huh JR, Seol JH, et al. Drosophila pink1 is required for mitochondrial function and interacts genetically with parkin. Nature. (2006) 441:1162-6. doi: 10.1038/nature04779

64. Park J, Lee SB, Lee S, Kim Y, Song S, Kim S, et al. Mitochondrial dysfunction in Drosophila PINK1 mutants is complemented by parkin. Nature. (2006) 441:1157-61. doi: 10.1038/nature04788

65. Hoshino A, Mita Y, Okawa Y, Ariyoshi M, Iwai-Kanai E, Ueyama T, et al. Cytosolic p53 inhibits Parkin-mediated mitophagy and promotes mitochondrial dysfunction in the mouse heart. Nat Commun. (2013) 4:2308. doi: $10.1038 /$ ncomms 3308

66. Woodall BP, Orogo AM, Najor RH, Cortez MQ, Moreno ER, Wang H, et al. Parkin does not prevent accelerated cardiac aging in mitochondrial DNA mutator mice. JCI Insight. (2019) 5:e127713. doi: 10.1172/jci.insight.127713

67. Kujoth GC, Hiona A, Pugh TD, Someya S, Panzer K, Wohlgemuth SE, et al. Mitochondrial DNA mutations, oxidative stress, and apoptosis in mammalian aging. Science. (2005) 309:481-4. doi: 10.1126/science.1112125

68. Dorn GW 2nd. Mitochondrial pruning by Nix and BNip3: an essential function for cardiac-expressed death factors. J Cardiovasc Transl Res. (2010) 3:374-83. doi: 10.1007/s12265-010-9174-x

69. Rana A, Oliveira MP, Khamoui AV, Aparicio R, Rera M, Rossiter HB, et al. Promoting Drp1-mediated mitochondrial fission in midlife prolongs healthy lifespan of Drosophila melanogaster. Nat Commun. (2017) 8:448. doi: 10.1038/s41467-017-00525-4

70. Frudd K, Burgoyne T, Burgoyne JR. Oxidation of Atg3 and Atg7 mediates inhibition of autophagy. Nat Commun. (2018) 9:95. doi: 10.1038/s41467-017-02352-z

71. Meng F, Yao D, Shi Y, Kabakoff J, Wu W, Reicher J, et al. Oxidation of the cysteine-rich regions of parkin perturbs its E3 ligase activity and contributes to protein aggregation. Mol Neurodegener. (2011) 6:34. doi: 10.1186/1750-1326-6-34

72. Wang C, Ko HS, Thomas B, Tsang F, Chew KC, Tay SP, et al. Stressinduced alterations in parkin solubility promote parkin aggregation and compromise parkin's protective function. Hum Mol Genet. (2005) 14:3885-97. doi: $10.1093 / \mathrm{hmg} / \mathrm{ddi} 413$

73. Rizza S, Cardaci S, Montagna C, Di Giacomo G, De Zio D, Bordi M, et al. S-nitrosylation drives cell senescence and aging in mammals by controlling mitochondrial dynamics and mitophagy. Proc Natl Acad Sci USA. (2018) 115:E3388-97. doi: 10.1073/pnas.1722452115

74. Oh CK, Sultan A, Platzer J, Dolatabadi N, Soldner F, McClatchy DB, et al. S-nitrosylation of PINK1 attenuates PINK1/parkin-dependent mitophagy in hiPSC-based Parkinson's disease models. Cell Rep. (2017) 21:2171-82. doi: $10.1016 /$ j.celrep.2017.10.068
75. Owada T, Yamauchi H, Saitoh SI, Miura S, Machii H, Takeishi Y. Resolution of mitochondrial oxidant stress improves aged-cardiovascular performance. Coron Artery Dis. (2017) 28:33-43. doi: 10.1097/MCA.0000000000 000434

76. Dai DF, Santana LF, Vermulst M, Tomazela DM, Emond MJ, MacCoss $\mathrm{MJ}$, et al. Overexpression of catalase targeted to mitochondria attenuates murine cardiac aging. Circulation. (2009) 119:2789-97. doi: 10.1161/CIRCULATIONAHA.108.822403

77. Latz E, Duewell P. NLRP3 inflammasome activation in inflammaging. Semin Immunol. (2018) 40:61-73. doi: 10.1016/j.smim.2018. 09.001

78. Youm YH, Grant RW, McCabe LR, Albarado DC, Nguyen KY, Ravussin A, et al. Canonical Nlrp3 inflammasome links systemic low-grade inflammation to functional decline in aging. Cell Metab. (2013) 18:519-32. doi: 10.1016/j.cmet.2013.09.010

79. Carmona-Gutierrez, Hughes AL, Madeo F, Ruckenstuhl C. The crucial impact of lysosomes in aging and longevity. Ageing Res Rev. (2016) 32:2-12. doi: 10.1016/j.arr.2016.04.009

80. Colacurcio DJ, Nixon RA. Disorders of lysosomal acidification-The emerging role of v-ATPase in aging and neurodegenerative disease. Ageing Res Rev. (2016) 32:75-88. doi: 10.1016/j.arr.2016.05.004

81. Hughes AL, Gottschling DE. An early age increase in vacuolar $\mathrm{pH}$ limits mitochondrial function and lifespan in yeast. Nature. (2012) 492:261-5. doi: 10.1038 /nature 11654

82. Folick, Oakley HD, Yu Y, Armstrong EH, Kumari M, Sanor L, et al. Aging. Lysosomal signaling molecules regulate longevity in Caenorhabditis elegans. Science. (2015) 347:83-6. doi: 10.1126/science. 1258857

83. Leeman DS, Hebestreit K, Ruetz T, Webb AE, McKay A, Pollina EA, et al. Lysosome activation clears aggregates and enhances quiescent neural stem cell activation during aging. Science. (2018) 359:1277-83. doi: $10.1126 /$ science.aag 3048

Conflict of Interest: The authors declare that the research was conducted in the absence of any commercial or financial relationships that could be construed as a potential conflict of interest.

Copyright $\odot 2020$ Liang and Gustafsson. This is an open-access article distributed under the terms of the Creative Commons Attribution License (CC BY). The use, distribution or reproduction in other forums is permitted, provided the original author(s) and the copyright owner(s) are credited and that the original publication in this journal is cited, in accordance with accepted academic practice. No use, distribution or reproduction is permitted which does not comply with these terms. 